\title{
Política Exterior Feminista: un análisis de la cooperación de Suecia
}

\author{
Bruna Soares De Aguiar*
}

\section{RESUMEN}

En 2014 Suecia fue el primer país en afirmar que pasaría a desarrollar una política exterior feminista (PEF). Este anuncio suscitó varias especulaciones sobre lo que significaría la inserción del paradigma feminista como política exterior. Autores y centros de estudio fueron, a lo largo de estos años, definiendo que una PEF trataría de un cambio en la ética de la política internacional revisando actores, estructuras y narrativas que tienden a generar una desigualdad de género y que suma opresiones de raza, identidad, etnia, religión, entre otros.

En este contexto, este estudio se considera importante al desarrollar un análisis en torno a la aplicación de la PEF sueca desde una perspectiva del Sur global, con el objetivo de observar las narrativas sobre las prácticas de cooperación sueca en el ámbito de la PEF. En este sentido, se ha optado por analizar la retórica sobre los resultados de la cooperación sueca en los tres primeros años de PEF de manera a observar si la narrativa sobre los resultados también presenta el mismo cambio propuesto por la definición de la cooperación, o si reproduce, en alguna medida, los discursos tradicionales. Este estudio fue realizado a partir del análisis de la narrativa presentada en documentos producidos por el gobierno sueco en el año de 2017.

Se puede concluir que, a pesar de que Suecia ha avanzado en el tema al integrar las demandas de los movimientos feministas a sus políticas de estado y gobierno, aún se puede observar la reproducción de discursos tradicionales de la cooperación internacional al desarrollo. Puesto que en su retórica no se explotan las asociaciones con los estados del Sur receptores de la cooperación y mantiene actores de la cooperación tradicional del Norte, reforzando el uso de la categoría género como indicador en la ayuda al desarrollo y poco cuestionadora de las relaciones de poder existentes entre Norte y Sur.

\section{Palabras clave}

Política exterior feminista; interseccionalidad; homogenización de las diferencias; solidaridad feminista; jerarquías.

\section{Tithe}

Feminist foreign policy: an analysis of Swedish cooperation

\section{Extended Abstract}

The announcement of Sweden's adherence to a feminist perspective on its foreign policy contributed to the gender agenda debate being broadened internationally. Therefore, it is considered important to analyze, from the South, how feminist foreign policy (FFP) has modified the discourses on traditional practices in foreign policy.

In order to carry out this study, the Swedish International Cooperation agenda was selected as a case to be examined.This research takes into account that the debate on gender and development has been articulated at least since the 1970s, and several contributions have underlined the need to question the power patterns involving the cooperation policies of the countries from North to South (Aguinaga et al, 20I I). Moreover, over the years countless alternatives to traditional and hierarchical practices of cooperation have been articulated by feminist women in both the South and the global North.

Given that Sweden adopted, as part of the feminist paradigm, the habit of carrying out a review of its policies, this study is developed from an analysis of Swedish rhetoric in the document on FFP policy practices released in 2017. Taking into account the narrative of seven themes, the extent to which there has been an insertion of the debates developed by Southern Feminisms in discourses on practices is debated.

DOI:

https://doi.org//0.15366/relacionesinternacionales2022.49.005

Formato de citación recomendado:

DE AGUIAR, Bruna SOARES (2022)."Política Exterior Feminista: un análisis de la cooperación de Suecia”,

Relaciones Internacionales, $\mathrm{n}^{\circ}$ 49, pp. 93-1 I0. 
In the first part of this article, a theoretical review is carried out on the debate around the construction of feminist solidarity in international politics (Mohanty, 2003;2008). It is understood that the category of women was included in the cooperation programs and policies through the process of homogenization of differences; that is, it was based on a universal assumption about feminist demands, without including the perspectives of the states receiving policies. Thus, a process of naturalization and generalization on the discursive performance and international practice on North-South cooperation was established. This has resulted in an elaboration on women of the North and South in opposite directions, in which there is an idea that one has to teach the other how to achieve gender equality. The argument of this study is that an FFP pursues the goal of building a shared relationship, in which cooperation is an interaction without hierarchies between the states involved; that is, there is a feminist solidarity in the construction of policies.

In other words, it seeks to identify the differences around gender issues and the category of women, adding them to political perspectives and thus developing more universal international approaches. While this should be an objective pursued by a FFP, and while there has also been discussion of what happens in traditional cooperation practices and discourses, northern countries tend to homogenize differences between women. This is done in line with neoliberal feminist perspectives, and does not include analyses of the patriarchal structure that promotes gender coloniality and generates subinclusive and superinclusive policies (Crenshaw, 2002).

In the second part of the article, the Swedish context that contributed to the elaboration of a paradigmatic feminist policy is presented. The country's women's social movements have had the capacity to articulate with the state over the years, which has accessed the welfare state and encouraged governments to assume discourses and policies that provide gender equality. The basis of this action is the formulation of the Swedish welfare state, which has elaborated domestically movement towards gender equality, and included social feminist demands in the formulation of public policies. In 20I4, this perspective was formally placed in international politics and, consequently, in the country's agendas, such as international development cooperation. With regard to this context of progress, the Swedish 2017 document was analyzed, seeking to identify elements that would point to a reproduction of the traditional perspective of cooperation in the country's rhetoric on the effectiveness of cooperation. This is where the actors of the North are the majority in the agreements, and where there is no discursive representation of the demands of the collectives of the South nor the processes of joint construction with the receiving countries.

In the study, it was possible to verify, like Nylund (2017), that the feminist foreign policy of Sweden produces totally feminist discourses, but also post-colonial rhetoric. In the feminist sense, we highlight the articulation capacity of feminism between the Swedish state and the feminist movements of the country in order to recognize, as in Llistar (2009), that when a country has the capacity to absorb the demands of social collectives in its international agendas -in the case of cooperation- it can be said that it is a cooperation of solidarity with low selfish interests. On the other hand, when we argue that Sweden has postcolonial discourses, we mean that, although it points out in its FFP manual that it seeks to develop a horizontal and intersectional policy, with the inclusion of local participation, in its rhetoric about the practice of cooperation the country does not emphasize joint actions with receiving countries. A narrative was also observed that values the performance of the state itself as a donor and its traditional partners in the North, such as development banks and private actors. Nevertheless, it does not present the integration of the critical vision of the southern feminisms on this classic performance of international cooperation.

In this way, it is argued that one side of feminist solidarity is missing. This means that, although there is recognition of the advance of Swedish feminisms in favoring the development of an FFP, the valorization and presentation of the performance of the southern actors is still lacking in the rhetoric in the results. So that, once again, they are not described as passive actors of cooperation, but that their different and critical perspectives contribute to the presentation of a more plural and universal discourse.

Finally, this article concludes that the development of critical analyses from the global South contributes to FFP being articulated in pursuit of the goal of feminist solidarity. We do not propose this analysis as a way to deny the advances established by Swedish politics, but to integrate the other part of feminist solidarity: including the vision of the South in the formulation of the agenda.

\section{KEYWORDS}

Feminist foreign policy; intersectionality; homogenization of differences; feminist solidarity; hierarchies. 
Los estudios de género comenzaron formalmente a ser desarrollados por medio de la producción académica en el Norte Global y, específicamente en las Relaciones Internacionales, las primeras contribuciones fueron de las feministas de la primera ola, en los países centrales, con la conocida pregunta de dónde estaban las mujeres en las Relaciones Internacionales (Tickner, 1992). A partir de esa pregunta, otras investigadoras fueron integrando el debate específico en el campo de las Relaciones Internacionales agregando cuestiones como la interseccionalidad, las condiciones materiales de las mujeres, los discursos sobre las mujeres del Sur y la colonialidad, por ejemplo. Sin embargo, a pesar del crecimiento en la variedad de la producción sobre la temática, todavía existe en el sistema internacional una cierta construcción narrativa tradicional de la perspectiva de género a partir de una genealogía que otorga los protagonismos a los esfuerzos desarrollados en los países del Norte.

De esta forma, este trabajo es un esfuerzo a partir del Sur Global de desarrollar una investigación feminista con el objetivo de destacar las perspectivas iniciales que introdujeron un debate sobre el cuestionamiento de los espacios institucionales internacionales y sus estructuras basadas en relaciones jerárquicas de género. Además, también busca examinar cómo este sistema ha absorbido las cuestiones más específicas involucradas en los debates del feminismo, como por ejemplo la raza, identidad, procedencia geográfica o clase social. Se parte así de la propuesta de construir un análisis de lo internacional por medio de perspectivas que visibilicen también las realidades del Sur Global en un sentido de identificación de las diferencias para establecer una acción conjunta y más universal en torno a políticas feministas (Mohanty, 2008).

Para ello, es importante cuestionar cómo las narrativas sobre las políticas de género están siendo desarrolladas por los países e instituciones en el sistema internacional y posteriormente internacionalizadas - si es por medio de un proceso más próximo a la exportación de políticas o por un intercambio entre socios, como preconizan los discursos horizontales adoptados. De este modo, sería posible comprender si incorporar el género como categoría en las políticas internacionales implica, por lo tanto, un cambio real del discurso que permite una comprensión más amplia de los conceptos y de los actores involucrados en las estructuras desiguales del sistema internacional.

Para alcanzar el objetivo establecido en el presente artículo, pretendemos discutir a partir del caso de Suecia en qué medida la concepción de la política exterior feminista (PEF) desarrolla su visión de cambio ético en las relaciones internacionales que favorece una integración amplia de las cuestiones de género como transversales en todas las agendas internacionales. Suecia es el caso analizado porque, en 2014 , fue el primer país en declarar que desarrollaría una política exterior basada en el paradigma feminista, apostando así por una posición internacional que procura integrar más ampliamente la agenda de género al más alto nivel de la relación entre los estados.

En política exterior, un ámbito particularmente importante en las relaciones del país nórdico con los países del sur es la cooperación al desarrollo. La agenda del desarrollo fue

El presente trabajo ha sido realizado con apoyo de la Coordinación de Perfeccionamiento de Personal de Nivel Superior - Brasil (CAPES) Código de Financiación 001 
ampliamente debatida por las feministas desde los años setenta, cuando comenzaron a cuestionar dónde estaban las mujeres en los proyectos de desarrollo. Con el paso del tiempo, se criticaron los modelos de desarrollo por no considerar las cuestiones de las desigualdades de género y también por no integrar a las mujeres en los procesos de toma de decisiones.Además, se señaló la importancia de debatir las relaciones de poder existentes en los debates de cooperación y desarrollo (Aguinaga et al., 20II). En este sentido, el sistema internacional ha realizado varias acciones para absorber estas demandas, y poco a poco se han creado instrumentos para estimular a los estados a incluir las cuestiones de género en sus políticas y prácticas de cooperación. Según López (2005), es posible observar un importante progreso a lo largo de los años en este tema, que ha permitido, al menos en la narrativa y en la retórica, que los gobiernos consideren la cuestión de la igualdad entre hombres y mujeres como objetivo y estrategia del desarrollo².

Por eso, buscamos analizar los discursos sobre las prácticas derivadas en la implementación de la PEF a partir de documentos producidos por el gobierno sueco y su Ministerio de Relaciones Exteriores. El objetivo es observar si además del discurso feminista, hay indicadores de reproducción de las jerarquías de poder de género. Estudiaremos cómo esta política de estado considera las más diversas experiencias de "ser" mujer. La propuesta principal de este texto es contribuir al debate sobre género en el área de las Relaciones Internacionales y, específicamente, centrarse en la reciente producción de investigaciones sobre la política exterior feminista desde el Sur.

\section{Las narrativas sobre el "ser mujer": origen de las políticas internacionales con perspectiva de género}

La construcción de "mujer" y "género" como categoría analítica atraviesa las distintas ondas del feminismo y la elaboración práctica, discursiva y política de esos movimientos alrededor del mundo. Cuando un grupo o institución se ocupa de la cuestión de género, se basa en una visión particular y específica de lo que se entiende por mujer.

Uno de los primeros textos de Chanda Mohanty (1988) es importante para comenzar la reflexión de ese estudio porque, según la autora, el tema de la mujer como categoría de análisis tradicionalmente remite a una idea de que las mujeres de todas partes, independientemente de la clase, localización cultural y etnicidad, sufren el mismo patrón de dominación masculina y experimentan las mismas opresiones, reduciendo a la mujer al estatus de víctima. Mohanty (1988) señaló que debido a esa idea hay una construcción de una categoría femenina basada en estereotipos, perdiendo así la visibilización de las diferentes maneras de ser mujer y quedando oscurecidas las experiencias individuales por un mito colectivo sobre el género femenino.

Es importante destacar también otra variable que contribuye a la construcción de mitos sobre las mujeres y su invisibilidad: el patriarcado. Esta estructura está formada por el poder político y económico que actúa sobre los cuerpos femeninos de manera que las mujeres están jerárquicamente subordinadas a los hombres. Puede añadirse además que el patriarcado como estructura tiene una relación intrínseca con el sistema capitalista y establece una división sexual

2 Este debate es mucho más amplio que el que presentamos en este estudio. Para profundizar sobre el tema se sugieren los trabajos de Caroline Moser, Idoye Zabala, Aguinaga et al., Irene Rodríguez Manzano y Vandana Shiva, entre otras. 
del trabajo en la que las mujeres son relegadas a los trabajos del campo privado y de la esfera del cuidado, y los hombres son responsables de las relaciones sociales y económicas en el ámbito público (Armstrong, 2020). En esta perspectiva, el patriarcado es una estructura que contribuye al mantenimiento del sistema capitalista (Armstrong, 2020).

Según Lugones (2020), esta estructura es anterior a los procesos de colonización, y en las colonias se sumaron patriarcados (de los colonizadores y el ya existente anteriormente), lo que generó un tipo de colonialidad de género que tiene relación con el eurocentrismo y la interseccionalidad raza/género. La cuestión de la colonialidad es importante porque junto con el patriarcado contribuye a una homogeneización de las diferencias, en el sentido de que articula visiones universales definiendo a quién necesita aprendizaje o transformación para alcanzar el modelo ideal del Norte. De acuerdo con Montanaro Mena (2016), incluso con el fin del colonialismo se mantiene la colonialidad que limita las identidades raciales, de género y políticas, resultando en un racismo estructural, sexismo e imperialismo. La colonialidad del poder se relaciona también con el patrón capitalista de poder que se estructura en relaciones de dominación entre sujetos sociales y disputa el control de dimensiones básicas de la vida, como el sexo, el trabajo y la autoridad colectiva. Según Lugones (2020), el poder capitalista y eurocéntrico es ejercido por dos ejes: la colonialidad del poder ${ }^{3}$ y la modernidad.

Estas dimensiones estructurales permiten comprender mejor la idea de homogeneización de las mujeres por medio de una categorización — que no es explicitada, pero está implícita en las narrativas políticas de "víctimas" y "héroes/heroínas". Esta realidad no permite una verdadera despatriarcalización de las políticas, ya que no cuestiona las estructuras que jerarquizan a las mujeres, sino que introduce las políticas de género en un espacio donde se desarrollan las violencias y jerarquías de género. Sin un cambio estructural del patriarcado y de la colonialidad, no es posible desarrollar políticas distintas de las hegemónicas, ya que implica solamente una inclusión de las mujeres en espacios estructuralmente patriarcales.

Además, es necesario analizar si la política exterior se basa exclusivamente en una internacionalización de las políticas del Norte hacia el Sur, lo que transmite la idea de aprendizaje y "salvación" por parte de los países del Norte. Para ello, es importante observar en qué medida se reproduce este patrón de dominación en la retórica sobre las prácticas de la Política Exterior Feminista. Según autoras como Mohanty (1988; 2003; 2008), Carneiro (2003), Paredes (20l4) y Parashar (20l8), la movilización de ese feminismo occidental blanco hegemónico sin actuar conjuntamente con los feminismos de los países destinatarios de las políticas puede reproducir jerarquías. Mohanty (1988) indica que en este patrón blanco del feminismo liberal las diferencias basadas en la biología son substituidas por la construcción cultural social. Hay un proceso de reduccionismo analítico que tiene consecuencias políticas directas. Tratar a la mujer como categoría "presocial” implica suposiciones sobre "quiénes son estas mujeres" a partir de las mujeres occidentales. Hay, por lo tanto, una esencialización de los problemas y deseos de las mujeres, a partir de la suposición de que debe haber una reconstrucción continua de la mujer del Sur,

3 De manera simplificada, la colonialidad del poder es una forma de dominación de las potencias del Centro en relación a los estados colonizados, basada en la construcción de diferencias de raza y género como forma de desarrollar una dominación económica y política, acompañada de dominación epistémica, lingüística, cultural, filosófica y científica. Ver González-Casanova (2006[1969]); Rivera Cusicanqui (2010); Quijano (2000); Escobar (2005). 
entendida siempre como víctima y oprimida, en contraste con la mujer occidental, independiente, moderna y liberal (Mohanty, 1988). En resumen, la creencia de que las mujeres forman un grupo homogéneo oprimido reduce la diversidad y complejidad de las circunstancias de las mujeres, lo que implica que hay un ejercicio de poder en estas representaciones y reproducciones.

Swati Parashar (2018) observa que puede haber, por parte de algunas prácticas políticas del Norte, una violencia epistémica y una marginación del Sur como contextos y espacios sin agencia, así como una complicidad feminista en promover agendas de estados sin cuestionar sus propios posicionamientos, compromisos éticos y privilegios. Existiría una cooptación de los feminismos por agendas neocoloniales y liberales que se concentran en la protección de las mujeres del Sur Global. Las diferencias no son solo entre las mujeres occidentales y no occidentales, sino también con respecto a las diferentes épocas y geografías. Cuando estos aspectos no son considerados, las agendas multilaterales endosan una visión específica de igualdad y paz que no tiene en cuenta todos los intereses y experiencias (Parashar, 2018), y que no suma las diferentes comprensiones ni construye perspectivas más solidarias de la política global.

Ante esto, retomamos la necesidad de establecer una solidaridad feminista básica, que cruce fronteras y no sea colonizadora (Mohanty, 2003). Las diferencias compartidas entre las feministas del Primer y Tercer Mundo serían herramientas para consolidar tal base solidaria (Mohanty, 2003; Matos, 2010), cuyo “[...] objetivo sería identificar, en las diferencias, los posibles vínculos y los rasgos compartidos que permitan la construcción de coaliciones y de solidaridad transversalizadoras" (Matos, 20I0, p. 77, traducción propia). Esta solidaridad puede ser una vía, junto con las políticas interseccionales, para romper con el patrón de universalización de las políticas exteriores.

La propuesta presentada por Chandra Mohanty (2003) entiende que los saberes de los pobres, indígenas y mujeres del llamado Tercer Mundo ofrecen una perspectiva más inclusiva del poder sistémico global. Este feminismo "sin fronteras" (Matos, 2010) acercaría el feminismo académico a las realidades globales que son atravesadas por género, raza y clase, de forma que no fueran reproducidas y repetidas las concepciones occidentales feministas. Se elaboraría así un feminismo localizado y de cuño anticapitalista y antiimperialista, con una valorización de las formas de resistencia colectiva de las mujeres en cualquier espacio geográfico y epistemológico (Mohanty, 2003).

Además, los estudios interseccionales destacan las diferentes marginaciones, desigualdades e injusticias en los ámbitos local, nacional e internacional que construyen y moldean las experiencias de inseguridad de las mujeres (Basu, 2016). Por consiguiente, la elaboración de políticas, sobre todo las feministas, debería tener en cuenta las numerosas inseguridades impuestas a las mujeres y a otras minorías, en particular en el Sur Global, por las acciones de los estados occidentales y sus prácticas intervencionistas neoliberales como forma de control de los estados descritos como débiles, y por la incidencia en las agendas de la sociedad civil local a través de donaciones y financiamiento de investigaciones (Parashar, 20I8).

La interseccionalidad puede ser definida como un concepto que busca reunir las consecuencias estructurales y dinámicas de la interacción de dos o más ejes de subordinación 
(Crenshaw, 2002).Además, “trata específicamente de la forma en que el racismo, el patriarcado, la opresión de clase y otros sistemas discriminatorios crean desigualdades básicas que estructuran las posiciones relativas de mujeres, razas, etnias, clases y otras" (Crenshaw, 2002, p. 177, traducción propia). Por eso, entendemos que las experiencias en los cruces de dominación son diferentes para las personas que están viviendo diversas condiciones. Cuando esta realidad no es tenida en cuenta por los feminismos, ciertos tipos de dominación pueden ser fortalecidos, y las mujeres de raza y clase dominante pueden reproducir las opresiones que denuncian en el patriarcado (Bernardes y Braga, 20I6).

La brasileña Sueli Carneiro (2003) también trató sobre esta cuestión al argumentar que el feminismo estaba preso de una visión eurocéntrica y universalizante de las mujeres, resultando en una imposibilidad de reconocimiento de las desigualdades que existen en el colectivo femenino: "Las voces silenciadas y los cuerpos estigmatizados de mujeres víctimas de otras formas de opresión más allá del sexismo, continuaron en silencio e invisibilidad” (Carneiro, 2003, p. II8, traducción propia).

Según Paredes (20I3), el proceso de liberalización de las políticas introdujo una concepción específica de la equidad de género, sustentada en la idea de que las luchas son las mismas. Paredes (20I3) explica que esta internacionalización de la equidad de género impacta las relaciones NorteSur, principalmente en la agenda de cooperación internacional, caracterizando las prácticas a partir de una estructura de subordinación que mantiene a los países del Sur Global en una constante dependencia de las políticas de ayuda al desarrollo promovidas por el Norte.

Para Kimberlé Crenshaw (2002), los enfoques del feminismo liberal tradicional comprenden las categorías de raza y género como marcas de la dominación, y por eso, las combaten a partir de la idea de la igualdad formal, o sea, con remedios legales. De esta forma, al desarrollar normas y políticas que delimiten la necesidad de equidad o empoderamiento, parece que los problemas de todas las mujeres en el mundo están en vías de solución. Sin embargo, Crenshaw (2002) señala que este tipo de práctica remite al siguiente "problema": el cruce de subordinación, que genera invisibilidades subinclusivas o superinclusivas. En la superinclusión la diferencia intragrupo es invisible y, "como consecuencia, las especificidades de los problemas dejan de ser consideradas y las políticas públicas orientadas al tema no son eficientes como deberían" (Bernardes y Braga, 2016, p. 723, traducción propia). En la subinclusión, la diferencia intragrupo es visible, pero se invisibiliza el conjunto de problemas generados por esta diferencia. Consecuentemente, "[...] la discusión estructural sobre la subinclusión y la superinclusión nos lleva al análisis de la dimensión política de las intersecciones” (Bernardes y Braga, 2016, p. 726, traducción propia).

Sobre la base de estas premisas, en nuestro análisis de la retórica sobre las prácticas de la política exterior feminista sueca tendremos en cuenta que ciertos discursos para la igualdad de género pueden promover una interpretación muy particular y homogénea de que todas las mujeres se enfrentan a los mismos desafíos y están incluidas en la misma categoría de vinculación, dando a entender que las soluciones pueden ser las mismas en todos los casos. Esta "homogeneización de la diferencia" es reproducida por los feminismos cuando se articulan en políticas limitándose a ofrecer remedios legales, sin actuar de manera conjunta con las perspectivas de los feminismos de los países receptores de una política de cooperación, por ejemplo.Además, esta realidad se amplía 
cuando hablamos de la política exterior de un país y principalmente de la agenda de cooperación, que como señala Paredes (20l3), sufre de una marca fundacional al comprender la ayuda como una escuela de los países del Norte en dirección a los países del Sur. En el caso de la agenda de género esta práctica de enseñanza - traducida en internacionalización de políticas públicas y concepciones de igualdad - puede actuar sobre el entrecruce de subordinaciones generadas por la interseccionalidad, con la consiguiente invisibilidad superinclusiva y las subinclusivas.

En suma, se debe tener en cuenta en qué medida la ética feminista es movilizada para reproducir determinados patrones de dominación, y cómo en muchos casos los feminismos son cómplices en la (re)producción de jerarquías — sean raciales, económicas, de poder, de géneroy trabajan de forma conjunta con los estados neoliberales en la promoción de una visión liberal de la igualdad de género y del empoderamiento (Parashar, 20 I8). Igualmente, debe considerarse si están trabajando en conjunto, a partir de la identificación de las diferencias, en la construcción de una solidaridad feminista en el interior de la política exterior feminista (Mohanty, 2003; 2008). No es una deslegitimación o negación de los avances políticos en el ámbito multilateral y en las prácticas de estados, sino un llamamiento a expandir la comprensión de la mujer como categoría analítica y, con eso, ampliar las agendas incorporando la diversidad de experiencias.

\section{Estudio de caso: discursos sobre las prácticas de la política exterior feminista sueca}

Suecia fue el primer país en considerar la adopción de la "F" (Aggestam y Rosamond, 2016), o sea, del feminismo como forma de calificar su política exterior. En 2014, la entonces Ministra de Relaciones Exteriores, Margot Wallstrom, anunció que la política que orientaría su cartera ministerial sería feminista, levantando especulaciones sobre el concepto y la práctica derivada. La consolidación se dio a través de la publicación del Handbook on Sweden's feminist foreign policy, que delimitó la forma en que el gobierno pasaría a introducir el feminismo en el Ministerio y en las políticas realizadas por el país en el ámbito nacional e internacional.

En el campo doméstico, el gobierno de Suecia actúa por lo menos desde 1970 en la promoción de políticas para la igualdad de género, partiendo del ideal de empoderamiento económico de las mujeres, junto a acciones del estado de bienestar social. Según Anette Borchorst y Birte Siim (2008), la apertura del Partido Social Demócrata contribuyó a la adopción de políticas redistributivas que ayudaron a los movimientos feministas a tener fuerza para conjugar estas políticas con la participación en el mercado de trabajo en las décadas de los setenta y ochenta. En casi todos los países escandinavos, esta política doméstica fue la base del state feminism, un feminismo institucional basado en la igualdad de género y las políticas sociales, así como en la incorporación de mujeres en las carteras importantes del estado de bienestar social (Hernes, 1987).

Christina Bergqirist et al. (2007), señalan que en Suecia el fortalecimiento simultáneo del movimiento feminista fuera del Parlamento y las políticas de igualdad de género del gobierno representan un efecto positivo del pensamiento feminista, porque el estado sirvió como un espacio y vehículo para el activismo feminista. Con esta tradición formativa del estado de bienestar social es posible que hoy en día exista una perspectiva feminista también en la política exterior del país. 
Esta realidad es resultado de una concepción de la política exterior como política pública, en el sentido de que sus formulaciones atraviesan caminos semejantes a las políticas domésticas, por lo que se debe prestar atención a las especificidades y diferentes realidades que serán contempladas en su aplicación (Pinheiro y Milani, 20I3).

McBride y Mazur (2010) trabajan específicamente con las cuestiones que contribuyen a que un estado tenga la perspectiva de género en sus acciones políticas.Y las reflexiones producidas nos sirven en la medida en que destacan la importancia también de los movimientos feministas de primera ola principalmente; en los estados del Norte, específicamente en Escandinavia-, que condujeron sus acciones de modo que tuvieron la capacidad de influenciar a sus gobiernos para que los estados también absorbieran el paradigma feminista como manera de pensar y construir políticas. McBride y Mazur (20l0) añaden que las bases del feminismo estatal están insertadas en la combinación entre los recursos de agencia y sus características estructurales, los recursos de los movimientos de mujeres, las características del entorno político y los elementos de apoyo de la izquierda.

En el gobierno iniciado por el ex primer ministro Stefan-Lofven, además de desarrollar políticas clasificadas como feministas, la mitad de los ministerios pasaron a ser liderados por mujeres, se amplió el presupuesto para políticas de igualdad de género, se expandió la integración de género en las agencias gubernamentales y, en 2018, se estableció la Agencia Sueca de Igualdad de Género para implementar esta nueva agenda política. La política exterior feminista forma parte de las iniciativas de un gobierno feminista que tiene objetivos y normas para implementar una estrategia nacional de diez años para prevenir la desigualdad de género. ${ }^{4}$

Suecia basa su actuación doméstica e internacional en el llamado "Principio de las Tres R" (Ministerio de Asuntos Exteriores Suecia, 2019):

- Derechos (rights, en inglés): tiene como objetivo la promoción plena del disfrute de los derechos humanos por parte de mujeres y niñas, teniendo como línea de acción el combate a todas las formas de violencia y discriminación que impiden la libertad de acción.

- Representación (representation, en inglés): a partir del reconocimiento de la necesidad de participación de niñas y mujeres en los procesos de toma de decisiones, busca el diálogo con representantes de mujeres en todos los niveles, incluida la sociedad civil.

- Recursos (resources, en inglés): que subraya que una perspectiva feminista de la Política Exterior debe garantizar recursos para la promoción de la igualdad de género y de oportunidades para todas las niñas y mujeres.

La política sueca aborda los temas de la alta política como espacios para garantizar la igualdad de género y los derechos universales como un modo de alcanzar otros objetivos como la paz, la seguridad y el desarrollo sostenible. Los métodos se conciben en tres carteras del Ministerio de Asuntos Exteriores sueco: política exterior y de seguridad, cooperación al desarrollo y política comercial. Por otro lado, el país apunta que busca aplicar una perspectiva interseccional que permita esclarecer las diferentes identidades que existen entre mujeres, hombres, niñas y niños, con diferentes niveles de influencia y condiciones de vida (Ministerio de Asuntos Exteriores Suecia, 2019, p. 39).

4 Estas acciones fueron formalizadas por el informe Power, goals and agency - a feminist policy for a gender equal future (20I6). 
En la cartera específica de la cooperación, la cuestión feminista ha contribuido a la adhesión de la agencia sueca de cooperación, SIDA (en inglés), del Plan for gender integration. Además, las políticas formuladas por la SIDA se centran en el cumplimiento de la Política Sueca para el Desarrollo Global y establecen cooperación bilateral con treinta y cinco países en África, Asia, América Latina y Europa. La agencia describe su trabajo a través del establecimiento de alianzas con otros actores, negando la implementación de una ayuda intervencionista. Los proyectos desarrollados con el apoyo de la agencia de cooperación giran en torno a cuatro perspectivas: a) derechos humanos, b) medio ambiente y clima, c) igualdad de género y d) conflictos. A lo largo de los años, la SIDA ha establecido la igualdad de género como una de las principales estrategias de acción que abarca diferentes ámbitos, como I) los derechos económicos de las niñas y las mujeres, 2) la participación política, 3) el fin de la violencia de género, 4) la educación de las niñas, 5) salud y derechos sexuales y reproductivos, 6) paz y seguridad y 7) integración de la igualdad de género en programas de desarrollo y de asistencia humanitaria. Para la ejecución de los proyectos, se estableció el Gender Toolbox, dividido en herramientas (tools), resúmenes (briefs) y visiones temáticas (thematic overviews) con grados de prioridades que cambian constantemente de acuerdo con la variación del contexto de desarrollo.

De acuerdo con Aggestan, Rosamond y Kronsell (20I8), una política exterior feminista debe buscar un distanciamiento de los discursos y prácticas de la política tradicional que están éticamente orientadas por principios jerárquicos. Aggestan y Rosamond (2016) señalan que la palabra feminismo no es solo un cambio de nomenclatura, sino una propuesta de acción para renegociar las jerarquías del poder de género que existen en la política internacional.Y para eso, entendemos que se debe incorporar una perspectiva interseccional que no homogeneice las diferencias. Lo que intentamos comprobar es si este cambio se verifica realmente en los discursos sobre las prácticas desarrolladas.

\section{I. La implementación de la política exterior feminista sueca}

Uno de los compromisos asumidos en la aplicación de la política exterior feminista sueca es la revisión periódica de las políticas ya desarrolladas. En el informe que evaluó los tres primeros años, Sweden's feminist foreign policy examples from three years of implementation (Ministry for Foreign Affairs Sweden, 2017), se destacan actividades en siete objetivos, que se analizan a continuación. Es importante subrayar que, a partir del discurso sueco en el documento, se mencionan cuestiones sobre sus prácticas y no sobre los efectos de las prácticas. En este trabajo, nos limitamos a observar cómo la retórica de la eficacia de la cooperación puede o no ser superinclusiva, subinclusivas, y cómo puede haber homogenización de las diferencias e internacionalización de los discursos de políticas de cooperación.

1) Pleno disfrute de los derechos humanos por todas las mujeres y niñas: Suecia informa de que trabaja en espacios multilaterales con acciones generales como la inclusión de cuestiones relativas a la salud reproductiva en el Consejo de Derechos Humanos de las Naciones Unidas, participando en las Comisiones de Igualdad de Género del Consejo Europeo y ayudando al Banco Mundial en la identificación y lucha contra las legislaciones discriminatorias. La Comisión también se ha comprometido a reforzar el Plan de Acción de la Unión Europea, presentándose como actor clave para promover la inclusión de las mujeres. Se trata de una 
actuación alineada con una concepción del feminismo que presta atención al ámbito institucional, con un énfasis en el aspecto legal de las desigualdades y sin presentar el debate en torno a cuestiones más estructurantes y sustantivas como el patriarcado y la interseccionalidad.Al igual que Aguinaga et al. (200I), comprendemos que en la agenda de desarrollo hubo una incorporación de género por medio de las instituciones, manteniendo en alguna medida a las mujeres subalternas al tejido institucional. Hay avances en la producción de indicadores que visibilicen las situaciones de las mujeres, pero no hay tanta atención en la cuestión de las relaciones de poder y de las estructuras patriarcales de esas instituciones.

2) Protección contra la violencia física, mental y sexual de todas las mujeres y niñas: Suecia informa que ha implementado un estudio en la República Democrática del Congo (RDC), con apoyo de la ONU Mujeres ${ }^{5}$, sobre normas masculinas, es decir, las costumbres que actúan directamente en la acción de las instituciones y de los sujetos desde una perspectiva de género. También indicó que actuó en Guatemala, en colaboración con la Fiscalía, para investigar casos de esclavitud sexual, y con UNICEF para prevenir el acoso infantil y el turismo sexual. También aquí es evidente el enfoque legalista, centrado en las normas internacionales de género. No es un cuestionamiento sobre la importancia de tratar cuestiones como el acoso infantil o la participación de mujeres en temas considerados como high politics, pero nos parece importante subrayar la idea de universalidad presente en la formación de la agenda internacional de género, que ofrece un paquete de temas que se tratan en todos los casos, independientemente del país o grupo de países beneficiarios de los acuerdos de cooperación. El uso de la estrategia Gender Toolbox en este ámbito es evidente. Aunque Suecia subraya que este método se aplica teniendo en cuenta los contextos locales, en su discurso sobre la implementación no indica cuáles eran las adaptaciones que se han hecho a partir de las realidades específicas de Guatemala o RDC, por ejemplo. Por otro lado, las investigaciones feministas críticas ya llamaban la atención sobre la acción a partir de un paquete de herramientas preestablecidas por una visión más tecnocrática del desarrollo, que puede, en algunos casos, indicar una transferencia colonial de configuraciones epistemológicas del Norte al Sur (Aguinaga et al., 20II).

3) Participación de las mujeres y las niñas en la prevención y resolución de conflictos y en la consolidación de la paz después de los conflictos: subraya el papel que ha desempeñado internacionalmente en la sensibilización sobre la violencia sexual como arma de guerra contra las mujeres, reforzando las capacidades de los países para actuar legalmente contra ese crimen, además de prestar asistencia a las víctimas. Toma nota asimismo de su impulso a la participación de las mujeres en los procesos de paz en Asia, África, América Latina y Oriente Próximo, y subraya que, mientras el país fue miembro del Consejo de Seguridad de las Naciones Unidas, la acción feminista fue una prioridad; así lo prueba la formación de mediación de mujeres en Afganistán, en cooperación con la Academia Folke Bernadotte, creando una red de mujeres para los procesos de paz con el objetivo de proporcionar herramientas a las afganas locales para que desempeñen un papel fundamental en los esfuerzos de consolidación de la paz del país ${ }^{6}$. Posteriormente, lanzó la Supplementary strategy for the

Suecia es el principal donante de ONU Mujeres, además de un socio estratégico para acciones de fortalecimiento de la igualdad de género a través del Strategic Partnership Framework 2017-2020.

6 Esta práctica siguió los principios adoptados en el Nordic Women Mediators, un proyecto compuesto por mujeres de países nórdicos con 
Folke Bernadotte Academy: Development cooperation and peace support operations in Afghanistan 2018-20 /9, que delimita los principios de las actividades a realizar.A pesar del uso de términos como "sinergia", “colaboración”, "perspectiva sensible del conflicto", no se explican de manera específica las actividades realizadas o de participación de las afganas en la toma de decisiones. Suecia es también el principal donante de la Unidad de Investigación y Evaluación de Afganistán (AREU) ${ }^{7}$, que se describe como un instituto independiente que tiene como misión informar e influenciar la política y la práctica, buscando la igualdad a partir del desarrollo de investigaciones. Sin duda, esta financiación tiene una influencia en las metodologías y concepciones adoptadas por esta organización. En su tesis, David Llistar (2009) argumentó que la financiación de las ONG en países que reciben cooperación contribuye a trasladar las concepciones e intereses de los países del Norte a los países del Sur, y que las organizaciones son limitadas en sus acciones en determinados temas.

4) Participación política e influencia de las mujeres y las niñas en todos los ámbitos de la sociedad: Suecia destaca su actuación como miembro del grupo director en la iniciativa International Gender Champions Geneva, una red de líderes de los estados miembros, de organizaciones internacionales y de la sociedad civil que trabajan por la igualdad de género, además de su trabajo con los bancos de desarrollo. Este punto es muy interesante para considerar las alternativas propuestas por los estados del Norte en términos de ayuda al desarrollo, dado que, junto con otros países escandinavos, Suecia supera los niveles de cooperación financiera de los países de la Organización de Cooperación y Desarrollo Económicos (OCDE). Además, Suecia tiene una posición más crítica con estados y organismos internacionales que tienen modelos de cooperación que persiguen intereses específicos, normalmente con países que sean sus colonias (LLISTAR, 2009). Suecia no tiene esta tradición colonizadora y por eso su actuación es distribuida por los países del Sur de manera general, pero incluso su apoyo a las instituciones internacionales de desarrollo es muy criticado por las feministas de los lugares que reciben cooperación. Por ello, como un análisis del feminismo del Sur, se entiende que es importante que en la retórica de la eficacia de la cooperación sueca se señalen también los cambios realizados por el feminismo del país en una acción de cooperación, desde su narrativa, pasando por las reflexiones de las estructuras de las acciones de los socios, hasta los resultados específicos.

5) Pleno disfrute de los derechos económicos y empoderamiento de todas las mujeres y niñas: Suecia señala que ha trabajado principalmente de conformidad con la Agenda 2030 y el Programa de Acción para la Financiación del Desarrollo de Addis Abeba. Además, destaca su cooperación con instituciones del Norte, como la OCDE, para incluir la igualdad de género en sus perspectivas. También financió una Conferencia en las Naciones Unidas en julio de 2017 para desarrollar herramientas de inclusión de la perspectiva de género en tratados comerciales.Y colabora con grandes empresas suecas, como H\&M y IF Metall, para

experiencia en procesos de construcción de paz, y que tiene como objetivos aumentar el número de mujeres nórdicas que están activamente involucradas en los esfuerzos internacionales de consolidación de la paz; apoyar a mediadoras no nórdicas y participar en iniciativas similares en otros países o regiones.

AREU también recibe fondos de la Unión Europea, Global Challenges Research Fund (GCRF), Fundación para la Promoción de la Sociedad Abierta (FPOS), el Instituto Médico Francés para la Madre y el Niño (FMIC), Royal United Services Institute (RUSI), Institute for Integrated Transitions (IFIT) y ONU Mujeres. 
promover un buen diálogo entre trabajadores y empleadores en Bangladesh, como parte de la iniciativa Global Deal ${ }^{8}$. Esta es la misma cuestión que abordamos en el punto anterior (4), en el sentido de iquiénes son los socios de la cooperación feminista? Porque tal como establece el discurso del Manual (Handbook) —donde Suecia subrayó un cambio de las prácticas internacionales a partir de concepciones no jerarquizadas de las relaciones de género, y con atención a la cuestión de la interseccionalidad-, es importante que no se reproduzcan reflexiones de perspectivas que ya han sido superadas, por ejemplo incluyendo en la retórica la categoría género, pero manteniendo la misma estructura. Esto fue exactamente lo que las feministas del Sur criticaron en los abordajes de Mujeres y Desarrollo de la década de los setenta, porque no estaban cuestionando para quién es el desarrollo, quién practica el desarrollo y con qué objetivo. De esta forma, resulta difícil integrar las visiones del Sur y sus contribuciones al desarrollo cuando se limitan a las instituciones del Norte como actores financieros que proporcionan la ayuda, generando una idea de transferencia de políticas con un mito del héroe que ayudará a las mujeres víctimas. Lo importante es que se considere a la hora de establecer alianzas lo que dicen las mujeres feministas del estado que van a recibir la ayuda, lo que piensan y cuáles son sus experiencias con los actores que la PEF está invitando a participar en la cooperación.

6) Salud sexual y reproductiva y derechos de las mujeres y niñas: Suecia destaca que coorganizó la Conferencia She Decides 9 (en Bruselas, 2017), como forma de promover universalmente los derechos sexuales y reproductivos de todas las niñas y mujeres. También realizó, en 2016, una donación de 5 millones de dólares para el desarrollo e implementación de un programa para transmitir conocimiento sobre salud reproductiva y aborto en Mozambique, destacando las capacidades decisorias de niñas y mujeres sobre sus cuerpos, además de informar sobre métodos anticonceptivos. Creó un programa de apoyo a la educación sexual en las escuelas de Zambia, en asociación con la UNESCO, que se difundió a otros países africanos. Y desarrolló un programa de obstetricia llamado Midwives4All Campaign en países como Afganistán, Zambia, Sudán del Sur y Myanmar.

7) Actividades internas del Servicio Exterior de Suecia para promover la política: centrado en la institucionalización de las prácticas feministas en el Servicio Exterior, destaca la comunicación y la diplomacia pública en cuestiones de género, el aumento del número de mujeres candidatas a puestos de dirección y la revisión de la evolución salarial como lucha contra la desigualdad entre hombres y mujeres, entre otros.

Todas estas acciones están pautadas en los principios de las Tres " $R$ " delimitadas en la política exterior feminista, y tienen como objetivo general llevar las buenas prácticas desarrolladas en el ámbito nacional a otros países. Esto genera la idea de una exportación del modelo sueco de igualdad de género que no profundiza en cómo se establecen las relaciones o cuáles son los métodos desarrollados junto a las mujeres de los estados receptores, lo que contribuye a reforzar la imagen más tradicional de la cooperación. El argumento defendido en esta investigación es que

\footnotetext{
Busca reunir a diversos interesados para promover soluciones conjuntas y un diálogo social eficaz con respeto y confianza mutuos que cree condiciones favorables a la colaboración entre empleadores, trabajadores y gobiernos. Según la web de la organización, estas prácticas pueden promover la competencia y mejorar la estabilidad económica y la prosperidad compartida.

9 Movimiento creado en 2017 en respuesta a las políticas conservadoras de Donald Trump con respecto a la salud reproductiva.
} 
este proceso de internacionalización de la política feminista debe ser observado más allá del ideal occidental de igualdad de género, incorporando la visión de los países receptores, sus discursos y demandas. Porque es una forma de crear una solidaridad feminista no solo en la política exterior de forma general, sino pensando en carteras centrales como la cooperación. Es importante resaltar que hay muchos trabajos desarrollados por feministas proponiendo alternativas al modelo de desarrollo actual, como la economía solidaria, la economía feminista — pensada primeramente por las feministas del Norte-, o el Buen Vivir en América Latina, por ejemplo.

En el marco de este análisis, se puede argumentar que falta la otra parte de la solidaridad feminista en el informe de prácticas de Suecia para que sea realmente posible identificar una construcción conjunta con los países beneficiarios. Si una PEF es un cambio ético, es importante que esta parte también esté representada en los documentos sobre los resultados, en la retórica de la eficacia de la ayuda. No es interesante que en una PEF la categoría del género sea mantenida solamente como indicadores del desarrollo para las organizaciones internacionales. Ahora que se ha dado un paso adelante, que las feministas han conquistado la integración de las lentes del género en la cooperación y han tenido éxito en llevar el feminismo como paradigma de una política exterior, es importante ir más allá.

\section{2. ¿Qué revela la retórica sobre las prácticas políticas suecas?}

El análisis realizado en este artículo puede ser complementado por la argumentación de Nylund (2017). La autora sugirió dos tipos ideales sobre los discursos de la política exterior feminista sueca: Gendered Poscolonial Discourse y Fully Feminist Discourse ${ }^{10}$, y concluyó que la política exterior feminista no puede ser colocada exclusivamente en ninguno de los dos tipos, ya que, aunque existe un discurso que reproduce relaciones desiguales de poder, algunas partes pueden considerarse totalmente feministas, transformando el discurso y contribuyendo al conocimiento sobre cómo evitar narrativas poscoloniales de género.

En relación con la perspectiva totalmente feminista de la política exterior feminista descrita por Nylund (2017), podemos argumentar que un factor que contribuye a esta realidad es la capacidad que tienen los movimientos feministas de Suecia de acceder al aparato estatal para influir en los procesos de construcción política. Como señala Llistar (2009), cuando un estado absorbe las demandas de los movimientos sociales para pensar y hacer su política de cooperación existe una indicación de que este país desarrolla un tipo de cooperación solidaria con bajos intereses egoístas. Sin embargo, también creemos que sigue siendo posible identificar las narrativas en torno a un discurso poscolonial de género y que genera en cierta medida una reproducción de la retórica tradicional de las políticas de desarrollo del Norte hacia el Sur —que se han debatido ampliamente durante décadas en los países del Sur como una reproducción de la lógica de la colonialidad de poder que también puede ser de género (Lugones, 2020). Y es este segundo sentido el que vamos a explorar en esta argumentación, ya que creemos en la necesidad de unir lo que ya es producido por los feminismos del Norte en la PEF con las contribuciones críticas desde el Sur — de los países receptores - en un intento de llevar la solidaridad feminista

\footnotetext{
10 Es decir, un discurso poscolonial de género y un discurso plenamente feminista, en un intento de comprender la dimensión de la narrativa de Suecia, analizando si la política exterior feminista toma o no en consideración jerarquías de género fomentadas por ideas coloniales, sin tener en cuenta la diversidad de experiencias femeninas.
} 
(Mohanty, 2003) a la política exterior femenina.

Los puntos destacados en la sección anterior demuestran que el discurso feminista occidental puede, en cierta medida, reforzar la importancia fundamental que la igualdad de género ha adquirido en la propia formación de la identidad nórdica, y que se moviliza como medio de oponerse a la realidad de otros países y regiones (Loftsdóttir et al., 20I2) a partir de un "modelo de igualdad de género". Esta es una característica que se puede observar, de manera general, en los discursos tradicionales de los países del Norte que utilizan el debate de género como estrategia difusora de valores occidentales y liberales en el sentido subrayado por Mohanty (1988), al construir una homogeneización de la diferencia a partir de la suposición del otro como una negación —"'no hombre", "no desarrollado"- otorgando significados antagónicos que resultan en una relación de víctima y héroe/heroína.

Otro punto relevante es que, a pesar de incluir en sus documentos oficiales una perspectiva interseccional de la política exterior feminista, no es posible vislumbrar tanto esta característica en la retórica sobre las prácticas presentadas como resultado de sus tres primeros años de implementación. Así, nos parece que el feminismo continúa realizando una universalización de las prácticas de igualdad, tal como señalaba Sueli Carneiro (2003).Además, implica una superinclusión (Crenshaw, 2002), ya que se delimita una línea de acción a seguir independientemente de los países con los que se coopera, lo que implica que ciertas cuestiones no son absorbidas por la estructura de género, invisibilizando las especificidades.

En este sentido, la política exterior de Suecia puede no implicar un cambio en los discursos jerárquicos de la política exterior, manteniendo estrategias centradas en una dimensión liberal y multilateral con especial énfasis en la igualdad de género en las instituciones, sin cuestionar las estructuras patriarcales y de colonialidad de género presentes en ellas. Las agencias multilaterales, bancos de desarrollo y organizaciones internacionales siguen un manual muy específico en temas de género, que no pretende cambiar la dinámica del capitalismo que subyuga a las mujeres, principalmente aquellas racializadas. La interseccionalidad está lejos de estos espacios y sus modelos de desarrollo han sido intensamente criticados por los feminismos, principalmente del Sur. Una alternativa a estas prácticas sería la inclusión de la solidaridad feminista defendida por Mohanty (2003), que implicaría comprender que las mujeres están presentes en la sociedad capitalista de manera desigual, a partir de variables que las jerarquizan. La subinclusión es evidente porque Suecia integra una narrativa de demanda universal a partir de la experiencia específica del feminismo del régimen internacional, y en su retórica no señala los momentos en que reflexiona sobre las críticas realizadas al modelo de desarrollo y sus impactos para otras realidades femeninas alrededor del mundo.

Esta incidencia se refleja también en la financiación de proyectos. Como explica Parashar (2018), las donaciones del Norte a los países del Sur y el financiamiento de investigaciones pueden ser una práctica de tercerización de una ideología específica. En cooperación, el formato de inversión financiera funciona como forma de implementar modelos de igualdad de género que no surgen necesariamente de demandas locales ni resultan en soluciones eficaces para las opresiones enfrentadas en el ámbito local de los receptores de "ayuda". Así, estas narrativas refuerzan prejuicios que fomentan jerarquías de poder, principalmente en lo que concierne a 
la agenda de género. Es importante avanzar en la construcción de una agenda colectiva, a partir de las diferencias y de las diversas posibilidades de construcción de paz por las experiencias culturales, nacionales, de raza, etnia y clase. En gran medida, las cuestiones específicas parecen ser absorbidas por la estructura de género de Suecia al no contemplar en su retórica documental la nacionalidad, religión y raza como parte de un todo, limitándolas a un problema específico de mujeres, resultando en políticas superinclusivas.

Las políticas de cooperación sueca, tal como se presentan en el informe, parecen seguir centradas en los procesos de ayuda a los países del Sur, mientras que la formulación de la agenda se mantiene junto con los países y organizaciones multilaterales con una visión supuestamente universal del feminismo. Los ejemplos que destacamos aquí revelan la construcción de narrativas con una única perspectiva respecto a las demandas a partir de un supuesto consenso común, que entendemos próximo al institucionalismo liberal. Una política exterior feminista que no rompa con patrones jerárquicos puede acarrear una instrumentalización acrítica de los derechos de las mujeres y de la igualdad de género (Parashar, 2018). La inclusión de la igualdad de género como principio rector de la política internacional constituye sin duda un progreso, pero hacerlo sin tener en cuenta el contexto de desigualdad estructural global puede hacer al feminismo cómplice de otras jerarquías raciales y culturales. Al movilizar el feminismo como narrativa, sin cuestionar la heterogeneidad de la categoría analítica mujer, los países del Norte y las organizaciones multilaterales dirigen sus políticas y prácticas, una vez más, como forma de llevar la civilidad a los países del Sur.

A este respecto se plantea la cuestión de hasta qué punto el gobierno sueco critica la desigualdad de género en el mundo, o si se trata de una exportación de su modelo doméstico como forma de cumplir los compromisos internacionales en la agenda de género. Sin duda es imprescindible aumentar la atención sobre la situación de las mujeres en conflictos armados, o sobre la muerte de niñas y mujeres por falta de salud reproductiva, pero la noción de "igualdad de género" desde un discurso feminista integral no puede basarse en un movimiento de enseñanza unilateral, sino que debe incluir una perspectiva crítica que integre las dimensiones estructurales y locales; de lo contrario, se implementarán otros patrones de poder y se construirán nuevas relaciones de dependencia.Y esto debe ser tenido en cuenta en sus reflexiones sobre la eficacia de las prácticas y en la presentación de los resultados.

La promoción de una política exterior feminista requiere distanciarse de los discursos y prácticas tradicionales de la élite internacional que están éticamente orientados por principios jerárquicos (Aggestam, Rosamond y Kronsell, 2018). Al adoptar una política exterior feminista, el estado se compromete con la inclusión de la perspectiva de las mujeres en la producción de sus políticas. ¿Pero, cómo hacerlo, teniendo en cuenta las estructuras de poder existentes?

\section{Conclusión}

Este artículo pretendía realizar un análisis del modelo de política exterior feminista implementado por Suecia desde una perspectiva feminista del Sur Global. Creemos que, por ser el primer país en incluir el paradigma feminista en la política exterior, Suecia ha fomentado el entusiasmo y el 
optimismo sobre sus discursos y prácticas. Sin embargo, aún son recientes los análisis sobre los impactos y las formas de cambios implementados por el país. No se ha pretendido disminuir los avances realizados por el gobierno sueco y por los colectivos feministas del país, sino llamar la atención y cuestionar los presupuestos de neutralidad de las relaciones internacionales que, en muchos casos, naturalizan ciertos patrones jerárquicos de cooperación. Por eso, se presta especial atención a los aspectos que fomentan la reproducción de patrones de poder a partir de una comprensión naturalizada del “otro” como sujeto que demanda ayuda, o sea, como víctima.

Una política exterior feminista con potencial transformador del orden patriarcal en el que estén insertadas las instituciones y prácticas internacionales incentiva la participación de la sociedad civil local en la elaboración de alternativas y, principalmente, en la construcción de la ética feminista, además de cambiar los actores tradicionales de la cooperación y de cuestionar las estructuras. Por ello, es importante que Suecia, en su retórica sobre los resultados de las prácticas, cuestione este régimen de género que tradicionalmente actúa en las políticas de cooperación, demostrando que lo que indican sus normativas sobre PEF y los compromisos nacionales de ampliación de la perspectiva del feminismo con los socios cooperantes de hecho se producen.

Es señalado por estudios feministas del Sur que la narrativa occidental liberal contribuye a fortalecer el prejuicio de que las mujeres del Sur son víctimas que demandan ayuda, y que necesitan formarse en las buenas prácticas del Norte para alcanzar la igualdad de género. Las jerarquías de poder se refuerzan cuando Suecia se coloca como agente que lleva las alternativas para acabar con las opresiones vividas y no presenta las críticas de los feminismos de los países beneficiarios y/o las acciones conjuntas en torno a una mayor comprensión de la igualdad de género. A ello hay que sumarle, en los informes sobre las prácticas, la ausencia de la perspectiva del Sur como grupo capaz de construir alternativas, o sea, como agentes activos y no solamente como receptores pasivos de las políticas.

En este sentido, mientras no haya una política exterior feminista crítica con las estructuras y éticas vigentes en el orden mundial, las formas de subordinación femenina serán mantenidas y reproducidas. Por eso, creemos que estudios como este son importantes en una búsqueda de la solidaridad feminista, pues es una indicación de dónde pueden mejorarse las retóricas para presentar una política exterior femenina que verdaderamente rompa con la tradición de la homogeneización de las diferencias de las mujeres del Sur y sea posible una solidaridad feminista.

\section{Referencias}

Aggestam, K.y Rosamond,A.B. (2016). Swedish feminist foreign policy in the making: ethics, politics, and gender. Cambridge University Press.

Aggestam, K., Rosamond, A.B. y Kronsell, A. (2018). Theorising feminist foreign policy. International Relations, I (33), 23-39.

Aguinaga, M., Lang, M., Mokrani, D. y Santillana,A. (20 I I). Pesar desde el feminismo: críticas y alternativas al desarrollo. En Lang, M. y Mokrani, D. (Eds.). Más allá del desarrollo (pp. 55-82). Fundación Rosa Luxemburgo.

Armstrong, E. (2020). Marxist and Socialism Feminism. Study of Women and Gender. Smith College.

Basu, S. (20I6). The Global South Writes I 325 (Too). International Political Science Review, 37 (3), 362-374.

Bernardes, M.N.y Braga, M.I.B. (20I6).Violências Interseccionais silenciadas em medidas protetivas de urgência. Direito \& Praxis, 7 (I5), 7 I5-740.

Borchorst, A. y Siim, B. (2008). Woman-friendly policies and state feminism: theorizing Scandinavian gender equality. Feminist Theory, 9(2), 207-224. 
Carneiro, S. (2003). Mulheres em movimento. Estudos Avançados, 17 (49), I I 7 - 132.

Crenshaw, K. (2002). Documento para o encontro de especialistas em aspecto da discriminação racial relativos ao gênero. Estudos Feministas, 10 (I), I7I- I88.

Hernes, H. (1987). Welfare state and woman power: essays in state feminism. Norwegian University Press.

Llistar, D. (2009). Anticooperación: interferencias globales Norte-Sur - los problemas del Sur no se resuelven con más ayuda internacional. Ediciones Icaria.

Loftsdóttir, K., Jensen, L. y Kershen, D.A.J. (20I2). Whiteness and postcolonialism in the Nordic region: exceptionalism, migrant others and national identities. Taylor and Francis.

López, I. (2005). Género en la agenda internacional del desarrollo: un enfoque en derechos humanos. Revista Académica de Relaciones Internacionales, 2, I-36.

Lugones, M. (2020). Colonialidade e gênero. En Hollanda, H. B.. Pensamento feminista hoje: perspectivas decoloniais. Bazar do Boitempo.

Matos, M. (2010). Movimento e teoria feminista: é possível reconstruir a teoria feminista a partir do Sul Global. Revista de Sociologia Política, 18 (36), 67-92.

Mcbride, D. y Mazur, A.G. (20I0). The politics of state feminism: innovation in comparative research. Temple University Press.

Ministerio de Asuntos Exteriores Suecia (2019). Manual política exterior feminista de Suecia. Government Offices of Sweden. Ministry of Foreign Affairs.

Ministry for Foreign Affairs Sweden (2017). Sweden's feminist foreign policy examples from three years of implementation. Government Offices of Sweden, Ministry of Foreign Affairs.

Mohanty, C.T. (1988). Under Western eyes: feminist scholarship and colonial discourses. Feminist Review, 30, 6I-88.

Mohanty, C.T. (2003). Feminism without borders: decolonizing theory, practicing solidarity. Duke University.

Mohanty, C.T. (2008). De vuelta a "bajo los ojos de Occidente": la solidaridad feminista a través de las luchas anticapitalistas. En Suárez Navaz, L. y Hernández, R. A. (Eds). Descolonizando el feminismo: teorías y prácticas desde los márgenes (pp. 407-458). Cátedra.

Montanaro Mena,A.M. (20I6). Hacia el feminismo decolonial en América Latina. En Blanco, M.y San Segundo, R. (Eds.). Investigación joven con perspectiva de género (336-355). Instituto de Estudios de Género. Universidad Carlos III de Madrid.

Nylund, M. (2017). A fully feminist foreign policy? A postcolonial feminist analysis of Sweden's feminist foreign policy. (Tesis doctoral). Uppsala University.

Parashar, S. (2018). The WPS agenda: a postcolonial critique. En Davies, S. y True, J. (Eds.). The Oxford handbook of women, peace, and security. Oxford Handbooks Online.

Paredes, J. (20I3). Hilando fino: desde el feminismo comunitario. Cooperativa El Rebozo.

Pinheiro, L. y Milani, C.R.S. (20I3). Política externa brasileira: os desafios de sua caracterização como política pública. Contexto Internacional, 35 (I), I I-4I.

Tickner, J.A. (1992). Gender in international relations: feminist perspectives on achieving global security. Columbia University Press. 


\section{RELACIONES INTERNACIONALES}

Revista académica cuatrimestral de publicación electrónica Grupo de Estudios de Relaciones Internacionales (GERI)

Universidad Autónoma de Madrid, España

https://revistas.uam.es/relacionesinternacionales

ISSN 1699 - 3950

f facebook.com/RelacionesInternacionales

3. twitter.com/RRInternacional

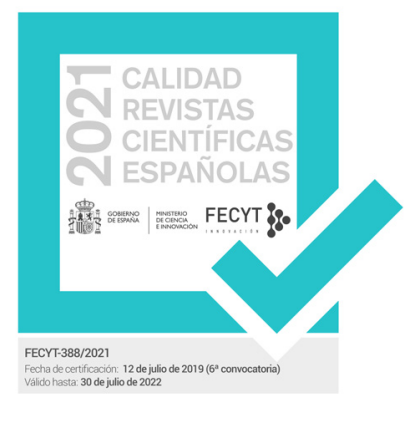

